INPLASY

PROTOCOL

To cite: Zhou et al. The efficacy and safety of Bismuth -containing quadruple therapy with different periods for Helicobacter pylori eradication treatment in China: a systemic review and network metaanalysis. Inplasy protocol 202230028. doi:

10.37766/inplasy2022.3.0028

Received: 07 March 2022

Published: 07 March 2022

Corresponding author:

Fang Zhou

fang.zhou1@takeda.com

Author Affiliation:

Takeda Pharmaceutical Company.

Support: Takeda

Pharmaceutical Company.

Review Stage at time of this submission: Completed but not published.

Conflicts of interest:

None declared.

\section{The efficacy and safety of Bismuth} -containing quadruple therapy with different periods for Helicobacter pylori eradication treatment in China: a systemic review and network meta-analysis

Zhou, F1; Xie, L².

Review question / Objective: To explore the efficacy and safety of quadruple therapy (Bismuth -containing quadruple therapy) with different periods for Chinese patients with Helicobacter Pylori (Hp)infection.

Eligibility criteria: We included Chinese Helicobacter pylori (Hp) patients who diagnosed of $\mathrm{Hp}$ infection based on one positive results on one or more of established techniques including Rapid Urease Test (RUT), Histology (with specific staining), Urease Breath Test (C13 or C14), or any molecular methods. Only diagnosed by serological measurements are not acceptable. There was no limitation on age and gender. Either newly diagnosed patients or treatment failure patients were included. Treatment failure patients were defined as a persistent $\mathrm{Hp}$ infection at least 4 weeks after completion of a first-line treatment. China includes mainland, Hong Kong, Macao, and Taiwan.

INPLASY registration number: This protocol was registered with the International Platform of Registered Systematic Review and Meta-Analysis Protocols (INPLASY) on 07 March 2022 and was last updated on 07 March 2022 (registration number INPLASY202230028).

\section{INTRODUCTION}

Review question / Objective: To explore the efficacy and safety of quadruple therapy (Bismuth -containing quadruple therapy) with different periods for Chinese patients with Helicobacter Pylori (Hp)infection.
Condition being studied: At present, the treatment periods of quadruple therapy are usually 7 days, 10 days, 14 days. But evidence for the best treatment periods is scant, thus we conducted a systematic review to summarize the efficacy and safety of quadruple therapy with different 
periods for Chinese patients with $\mathrm{Hp}$ infection.

\section{METHODS}

Search strategy: We conducted a systematic search in PubMed, EMBASE, the Cochrane Library, CNKI, Wanfang, and CBM on 3rd September, 2021, with limitation on China. All the eligible RCTs from 2000 to 2021 were included. The electronic search will be supplemented by hand-searching of reference lists of relevant systematic reviews at full-text screening stage to identify any additional published or unpublished material (grey literature) not retrieved by the electronic search.

1. PubMed: \#1. "Helicobacter Infections"[Mesh] OR "Helicobacter pylori" [Mesh] OR Helicobacter[tiab] OR Campylobacter[tiab].

\#2. ("controlled clinical trial"[pt] OR "Controlled Clinical Trials as Topic"[MeSH] OR "Random Allocation"[MeSH] OR "Double-Blind Method"[MeSH] OR "singleblind method "[MeSH] OR "Control Groups" [MeSH] OR "cross-over studies"[MeSH] OR random*[tiab] OR placebo[tiab] OR trial[tiab] OR groups[tiab] OR crossover[tiab] OR cross-over[tiab]) NOT ("Animals" [Mesh] NOT ("Humans"[Mesh] AND "Animals"[Mesh])). \#3. \#1 AND \#2.

\#4. "China"[Mesh] OR China OR Chinese OR Taiwan OR "Hongkong" OR Hongkong OR Macau OR Macao OR Beijing OR Shanghai OR Tianjin OR Chongqing OR "Inner Mongolia" OR Tibet OR Guangxi OR Sinkiang OR Ningxia OR Xinjiang OR Hebei OR Shanxi OR Liaoning OR Jilin OR Heilongjiang OR Jiangsu OR Zhejiang OR Anhui OR Fujian OR Jiangxi OR Shandong OR Henan OR Hubei OR Hunan OR Guangdong OR Hainan OR Sichuan OR Guizhou OR Yunnan OR Shaanxi OR Gansu OR Qinghai.

\#5. \#3 and \#4.

2. EMBASE: \#1. exp Helicobacter infection/ OR exp Helicobacter pylori" [Mesh] OR Helicobacter[tiab] Campylobacter.ab,ti,kw. \#2. (exp controlled clinical trial/ OR exp "Controlled Clinical Trial (Topic)"/ OR double blind procedure/ OR control group/
OR crossover procedure/ OR single blind procedure/ OR triple blind procedure/ OR placebo/ OR exp randomization/ OR (random* OR trial OR groups OR placebo* OR crossover OR "cross-over").ab, ti,kw.) AND exp human/

\#3. \#1 AND \#2.

\#4. exp China/ OR (China OR Chinese OR Taiwan OR "Hong kong" OR Hongkong OR Macau OR Macao OR Beijing OR Shanghai OR Tianjin OR Chongqing OR "Inner Mongolia" OR Tibet OR Guangxi OR Sinkiang OR Ningxia OR Xinjiang OR Hebei OR Shanxi OR Liaoning OR Jilin OR Heilongjiang OR Jiangsu OR Zhejiang OR Anhui OR Fujian OR Jiangxi OR Shandong OR Henan OR Hubei OR Hunan OR Guangdong OR Hainan OR Sichuan OR Guizhou OR Yunnan OR Shaanxi OR Gansu OR Qinghai).ti,ab,kw,ad,cp.

\#5. \#3 AND \#4.

3. The Cochrane Library: \#1 MeSH descriptor: [Helicobacter Infections] explode all trees.

\#2 MeSH descriptor: [Helicobacter pylori] explode all trees.

\#3 Helicobacter OR Campylobacter. \#4 \#1 OR \#2 OR \#3.

\#5 China OR Chinese OR Taiwan OR "Hongkong" OR Hongkong OR Macau OR Macao OR Beijing OR Shanghai OR Tianjin OR Chongqing OR "Inner Mongolia" OR Tibet OR Guangxi OR Sinkiang OR Ningxia OR Xinjiang OR Hebei OR Shanxi OR Liaoning OR Jilin OR Heilongjiang OR Jiangsu OR Zhejiang OR Anhui OR Fujian OR Jiangxi OR Shandong OR Henan OR Hubei OR Hunan OR Guangdong OR Hainan OR Sichuan OR Guizhou OR Yunnan OR Shaanxi OR Gansu OR Qinghai 110277. \#6 \#4 and \#5.

4. CNKI: (SU\%=(幽门螺杆菌+幽门螺旋杆菌 +幽门螺旋菌)^感染+hp感染+HP阳性+螺旋菌阳 性+螺杆菌阳性+螺旋菌感染+螺杆菌感染 OR TKA =(幽门螺杆菌+幽门螺旋杆菌+幽门螺旋 菌)^感染+hp感染+HP阳性+螺旋菌阳性+螺杆菌 阳性+螺旋菌感染+螺杆菌感染) AND (SU\%=随 机+盲法+双盲+单盲+三盲+交叉+RCT OR T K $A=$ 随机+盲法+双盲+单盲+三盲+交叉+RCT).

5. Wangfang: 主题:(("幽门螺杆菌" OR "幽门 螺旋杆菌" OR "幽门螺旋菌") AND "感染") OR 
"hp感染" OR "HP阳性" OR "螺旋菌阳性" OR "螺杆菌阳性" OR "螺旋菌感染" OR "螺杆菌感 染") and 主题:("随机" OR "盲法" OR "双盲" OR "单盲" OR "三盲" OR "交叉" OR "RCT").CBM

6. CBM: (("幽门螺杆菌"[不加权:扩展] OR "幽 门螺杆菌"[常用字段:智能] OR "幽门螺旋杆菌 "[常用字段:智能] OR "幽门螺旋菌"[常用字段: 智能]) AND "感染"[常用字段:智能]) OR ("hp 感染"[常用字段:智能] OR "HP阳性"[常用字段: 智能] OR "螺旋菌阳性"[常用字段:智能] OR "螺 杆菌阳性"[常用字段:智能] OR "螺旋菌感染"[常 用字段:智能] OR "螺杆菌感染"[常用字段:智 能]) AND ("临床对照试验"[不加权:扩展] OR " 临床对照试验(主题)"[不加权:扩展] OR "随机对 照试验"[不加权:扩展] OR "随机对照试验(主 题)"[不加权:扩展] OR "非随机对照试验(主 题)"[不加权:扩展] OR "随机"[常用字段:智 能]OR "盲法"[常用字段:智能] OR "双盲"[常用 字段:智能] OR "单盲"[常用字段:智能] OR "三 盲"[常用字段:智能] OR "交叉"[常用字段:智能] OR "RCT"[常用字段:智能]).

Participant or population: Chinese Helicobacter pylori (Hp) patients. Diagnostic methods limited to Rapid Urease Test (RUT), Histology (with specific staining), Urease Breath Test (C13 or C14), or any molecular methods but not serological measurements. There will be no limitation on age and gender. Either newly diagnosed patients or treatment failure patients will be included. A persistent $\mathrm{Hp}$ infection at least 4 weeks after completion of a first-line treatment was defined as treatment failure. China includes mainland, Hong Kong, Macao, and Taiwan.

Intervention: Different treatment duration of quadruple therapy (Bismuth -containing quadruple therapy), such as 7 days, 10 days and 14 days.

Comparator: Different treatment duration of quadruple therapy (Bismuth -containing quadruple therapy), such as 7 days, 10 days and 14 days.
Study designs to be included: Randomized controlled trials (RCTs).

Eligibility criteria: We included Chinese Helicobacter pylori $(\mathrm{Hp})$ patients who diagnosed of $\mathrm{Hp}$ infection based on one positive results on one or more of established techniques including Rapid Urease Test (RUT), Histology (with specific staining), Urease Breath Test (C13 or C14), or any molecular methods. Only diagnosed by serological measurements are not acceptable. There was no limitation on age and gender. Either newly diagnosed patients or treatment failure patients were included. Treatment failure patients were defined as a persistent $\mathrm{Hp}$ infection at least 4 weeks after completion of a first-line treatment. China includes mainland, Hong Kong, Macao, and Taiwan.

Information sources: We conducted a systematic search in the databases of PubMed, EMBASE, The Cochrane Library, CNKI. Wangfang and CBM. The electronic search will be supplemented by hand searching of reference lists of relevant systematic reviews at full-text screening stage. This is intended to identify any additional published or unpublished material (grey literature) not retrieved by the electronic search.

Main outcome(s): Primary outcome: Eradication rate at end of study (last follow-up timepoint), defined by original studies. Secondary outcome: 1. Adverse events, including total adverse events and specific adverse events. 2. Compliance, defined by original studies. If intention-totreat (ITT) population is available in original studies, we will select outcome data from ITT population. If ITT population is not available, we will use data derived from per-protocol (PP) population.

Quality assessment / Risk of bias analysis: We performed quality assessment according to criteria for assessment of risk of bias recommended by the Cochrane 2019 (e.g. the Cochrane Risk of bias tool for RCTs). 
Strategy of data synthesis: We used Network meta-analysis for this systematic review. For direct treatment comparisons, we conducted pairwise meta-analyses by synthesizing studies that compared the same interventions using a random-effects model that contained two or more studies. We calculated comparative effect sizes as odds ratios (RRs) with their 95\% confidence intervals (CIs). The network meta-analysis was based on the R Studio 4.0 Bayesian random effects model. The effect size of the network results was statistically realized based on odds ratio (OR) and 95\% confidence interval (CI). The consistency test is implemented based on the Chi test to evaluate whether there is a difference between the evidence of direct comparison and indirect comparison in the mixed effect that affects the authenticity of the network meta-analysis. In the analysis of this model, the overall consistency is tested first, and then the local inconsistencies between direct and indirect comparisons are tested using the node split method. Network results were presented, as well as the results of the intervention compared directly with placebo.

Subgroup analysis: Subgroup analysis was applied only for primary outcome. To explore the effect of the age, we planned to perform a subgroup analysis dividing the RCTs into 2 groups, children (aged younger than 18), adults (aged older than 18). To explore the effect of the eradication regimens of refractory Helicobacter Pylori infection, we planned to perform a subgroup analysis dividing the RCTs into 2 groups, treatment-naïve, rescue treatment.

Sensitivity analysis: We did not perform sensitivity analysis.

Language: No limited.

Country(ies) involved: China.

Keywords: Helicobacter Pylori (Hp); Chinese population; Network metaanalysis.
Contributions of each author:

Author 1 - Fang Zhou have involved in the conception of this study, designed it, and will participate in data interpretation.

Email: fang.zhou1@takeda.com

Author 2 - Li Xie have involved in the conception of this study, designed it, and will participate in data interpretation.

Email: li.xie@takeda.com 